\title{
Long-range correlation energies calculations for $\pi$ electronic systems
}

\author{
Hua Zhao \\ Institute of Condensed Matter Physics and Department of Physics, \\ ChongQing University, ChongQing, 400044, P.R.China
}

$2006 / 5 / 26$

\begin{abstract}
A simple formula for correlation energy $E_{c}$ of the $\pi$ electron systems is obtained under an approximation for the electronelectron interactions. This formula is related directly to square of the bond order matrix and the nearest-neighbor Coulomb electron-electron interaction. The influence of the correlation energy on the band energy gap is discussed. The values of the correlation energy for polyacetylene (PA) are calculated and can be compared with those for PA obtained by other methods, including ab initio method.
\end{abstract}

PACS number: $31.25 \mathrm{Qm}$

\section{Introduction}

The electron correlations have been a very important issue in investigating the electronic structures of various electron systems. Especially the electron correlations have a strong influence on the bonding properties of atoms and semiconductor band gap ${ }^{1}$. Hbwever, it is well known that it is very hard to completely solve a many electron system analytically using a single approximation because of the exchange and correlation problems. Hartree-Fock approximation deals with the exchange problem between the Same spins among electrons but not resolve the correlation between the opposite spins among electrons ${ }^{2,3}$. The local density approximation (LDA) of Kohn and Sham ${ }^{4}$ and later the density functional theory of Hohenberg and Kohn ${ }^{5}$ made a contribution (40) the exchange-correlation energy, denoted by $E_{e x}[\rho(\vec{r})]$ using a complicated functional form. In the LDA, the ground state exchange energy $E_{x}$ can be expressed an integral of the charge density. However, it is difficult to express the correlation energy E in an available form of integral of the charge density which is easy to calculate and only could be expressed as a numerical formula with the parameter $r_{s}$ after a large number of works ${ }^{6}$.

ㄱ Besides the LDA, there are other methods which can be used to deal with the correlation effects of various electron systems such as metal and nonmetal atoms, small and big molecules, as well as polymers. They include unrestricted self-consistency Field (SCF) Approximation ${ }^{3}$, Configuration Interaction method(CI $)^{7}$, coupled-cluster method ${ }^{8,9}$ that are applied to quantum chemistry and nuclear physics ${ }^{10}$, Jastrow wave-function method ${ }^{11}$ that is used to describe correlations in homogeneous fermion sfstems like the electron gas or liquid He-III ${ }^{12,13}$, the Projection Technique ${ }^{14}$ for the strongly correlated systems, and the GWapproximation (the one-particle Green's function plus the screened Coulomb interactions) by Hedin ${ }^{15}$. For the conjugated polymers such as polyacetylene $(\mathrm{CH})_{x}(\mathrm{PA})$ or poly $(p$-phenylenevinylene) (PPV), the exchange-correlation energies has been . Calculated by the method of the ab initio with GW-approximation ${ }^{16}$. For the various metal and nonmetal atoms, one used atomic Bethe-Goldstone equation under Hartree-Fock functions to calculate the electron correlation energies for their ground states ${ }^{17,18,19}$. For small or medium-size molecules(hydrocarbon molecules), a semi-empirical SCF scheme plus CNDO or INDO approximations was used to calculate inter-atomic correlations and intra-atomic correlations whose results could be compared with ab initio method ${ }^{20}$.

However, usually the discussion about the correlation energy concentrates mostly on the on-site Coulomb interaction, that is, from the term $n_{i \uparrow} n_{i \downarrow}$ and seldom specially on the lang-range Coulomb interaction, at least, on the nearest-neighbor Coulomb interaction. For strongly correlated systems, the on-site Hubbard interaction $U$ is much bigger than the nearest-neighbor Coulomb interaction $v$, so the contribution of the correlation energy are mainly from $U$. But it is well known that in the most conjugated polymers, the on-site electron-electron interaction $U$ is not so bigger than the nearest-neighbor interaction $v$ because of screening ${ }^{21}$.

In the past, people treated with the correlation energies both from the on-site Hubbard interaction $U$ and the long-range Coulomb interaction, In Ref.22, authors studied the correlation energies of polyethylene $\left(\mathrm{CH}_{2}\right)_{x}(\mathrm{PE})$ using the local ansatz ${ }^{22}$. In Ref.23, authors used the Gutzwiller ansatz as the variational ground state and studied correlation energy of polyacetylene (PA). First they used the Hubbard term plus SSH Hamiltonian to discuss the correlation energy and later added the nearest-neighbor interaction to get an effective $U_{\text {eff }}$ and discuss the correlation energy again. In Ref.24, author used a variational method ${ }^{25,26}$ to

${ }^{1}$ E-mail:huazhao@cqu.edu.cn 
study the correlation of PA in the PPP model where both Hubbard term and the long-range Coulomb interaction were included. But author did not specifically deal with the correlation energy from the long-range Coulomb interaction. It is important for the long-range interaction to calculate the band energy gap in the conjugated polymers ${ }^{27}$.

Although there were those studies above about the correlation energy both from the on-site Hubbard interaction $U$ and the long-range Coulomb interaction and people have been known the importance of the correlation between two $\pi$ electrons in understanding electronic properties of the conjugated polymers, such as the optical band gap, exciton excitation energy and exciton binding energy, polaron, etc., there is still lack of special studies on the correlation energy mainly from the long-range Coulomb interaction. Therefore, study of the correlation effect due to the Coulomb interaction of two adjacent $\pi$ electrons in the conjugated polymers becomes significant.

The purpose of the present work is to study the correlation energy mainly from the long-range Coulomb interaction (the nearest-neighbor interaction in this study). The starting point in this paper is the exchange-correlation energy $E_{e x}$. Although the correlation energy may be expressed as $E_{c}=\int \epsilon_{c} n(\vec{r}) d \vec{r}, \epsilon_{c}$ is hard to know and hard to obtain. Thus, available approximations will be adopted in this study. Finally, a formula of the electron correlation energy due to the nearest-neighbor Coulomb interaction is obtained in an analytical form. Using this formula, it is simple to calculated the correlation energy of a $\pi$ conjugated polymer and available to discuss the influence of the correlation energy on the band energy gap.

The arrangement of this paper is as follows. In section II, the expression of the pair-distribution function for the conjugated polymer systems by omitting wave function overlap integrals between two adjacent atomic sites is presented. In section III, an approximation for the electron-electron interaction integral is made and an analytical expression of the correlation energy for the conjugated polymers is obtained. In section IV, the correlation energy calculation for one-dimensional polyacetylene (PA) chain under tight-bind approximation (SSH Hamiltonian plus electron-electron interactions), and results are presented, and in section $\mathrm{V}$ is discussion with a summary.

\section{Pair-distribution function for electron systems}

The so-called correlation is the correlation hole appearing around an electron moving in the medium. Remarkably the correlation in fact is due to the electron-electron interaction among electrons. In a homogeneous or an inhomogeneous electron gas, the density operator of the electrons is expressed as a delta function $\hat{\rho}(\vec{r})=\sum_{i} \delta\left(\vec{r}-\vec{r}_{i}\right)$, the density distribution function of the electrons is the average of the operator over a given state $\mid \Phi>, \rho(\vec{r})=\langle\Phi|\hat{\rho}(\vec{r})| \Phi>$. The two-point density-density correlation function at a given state $\mid \Phi>$ is defined as

$$
\begin{aligned}
C\left(\vec{r}, \overrightarrow{r^{\prime}}\right) & =<\Phi\left|\sum_{i \neq j} \delta\left(\overrightarrow{r^{\prime}}-\vec{r}_{i}\right) \delta\left(\vec{r}-\vec{r}_{j}\right)\right| \Phi> \\
& \equiv<\hat{\rho}(\vec{r}) \hat{\rho}\left(\overrightarrow{r^{\prime}}\right)>
\end{aligned}
$$

where $\langle\ldots\rangle$ means $\langle\Phi|\ldots| \Phi\rangle$, the average of the operators over the ground states. To calculate the two-point densitydensity correlation function, one introduces the pair-distribution function which is defined $\operatorname{as}^{28}$

$$
<\hat{\rho}(\vec{r}) \hat{\rho}\left(\overrightarrow{r^{\prime}}\right)>=g\left(\vec{r}, \overrightarrow{r^{\prime}}\right)<\hat{\rho}(\vec{r})><\hat{\rho}\left(\overrightarrow{r^{\prime}}\right)>
$$

About the pair-distribution function $g\left(\vec{r}, \overrightarrow{r^{\prime}}\right)$ there are different approximations. For example it can be cast into an analytic expression for the homogeneous gas ${ }^{29}$. For a many-electron system, the density distribution $\rho(\vec{r})$ of the electrons can be expressed by

$$
\begin{aligned}
\rho(\vec{r}) & =2 \sum_{\mu}^{o c c}\left|\psi_{\mu}(\vec{r})\right|^{2} \\
& =\sum_{i j}^{N} \rho_{i j} \phi_{i}^{*}(\vec{r}) \phi_{j}(\vec{r})
\end{aligned}
$$

where the index $\mu$ refers to the occupied molecular orbital, $\mu=1,2, \cdots, N / 2$. The indices $i$ and $j$ refer to the atomic sites. $\phi_{i}(\vec{r})$ is the electron orbital wave function at the atomic site $i$ and is normalized to one. Here $\rho_{i j}=\sum_{\sigma}<a_{i \sigma}^{\dagger} a_{j \sigma}>$ where $a_{i \sigma}^{\dagger}\left(a_{j \sigma}\right)$ is the electron creative (annihilation) operator with spin $\sigma$ at the site $i(j) . \rho_{i j}$ is called the bond-order matrix ${ }^{28}$. Comparing (3) with $\rho(\vec{r})=<\Phi|\hat{\rho}(\vec{r})| \Phi>$, we can see that the density operator for a many-electron system can be represented by

$$
\hat{\rho}(\vec{r})=\sum_{i, j}^{N} \hat{\rho}_{i j} \phi_{i}^{*}(\vec{r}) \phi_{j}(\vec{r})
$$

with $\hat{\rho}_{i j}=\sum_{\sigma} a_{i \sigma}^{\dagger} a_{j \sigma}$. Then the two-point density-density correlation function is expressed as

$$
<\hat{\rho}(\vec{r}) \hat{\rho}\left(\overrightarrow{r^{\prime}}\right)>=\sum_{\sigma \sigma^{\prime}} \sum_{i j}^{N} \sum_{k l}^{N}<\hat{\rho}_{i j}^{\sigma} \phi_{i}^{*}(\vec{r}) \phi_{j}(\vec{r}) \hat{\rho}_{k l}^{\sigma^{\prime}} \phi_{k}^{*}\left(\overrightarrow{r^{\prime}}\right) \phi_{l}\left(\overrightarrow{r^{\prime}}\right)>
$$




$$
\begin{aligned}
& =\sum_{\sigma \sigma^{\prime}} \sum_{i k(i \neq k)}^{N}<\hat{\rho}_{i i}^{\sigma} \hat{\rho}_{k k}^{\sigma^{\prime}}>\left|\phi_{i}(\vec{r})\right|^{2}\left|\phi_{k}\left(\overrightarrow{r^{\prime}}\right)\right|^{2} \\
& +\sum_{\sigma \sigma^{\prime}} \sum_{i j(i \neq j)}^{N} \sum_{k l(k \neq l)}^{N}<\hat{\rho}_{i j}^{\sigma} \hat{\rho}_{k l}^{\sigma^{\prime}}>\phi_{i}^{*}(\vec{r}) \phi_{j}(\vec{r}) \phi_{k}^{*}\left(\overrightarrow{r^{\prime}}\right) \phi_{l}\left(\overrightarrow{r^{\prime}}\right)
\end{aligned}
$$

and

$$
\begin{aligned}
<\hat{\rho}(\vec{r})> & =<\sum_{i j \sigma}^{N} \hat{\rho}_{i j}^{\sigma} \phi_{i}^{*}(\vec{r}) \phi_{j}(\vec{r})> \\
& =\sum_{\sigma i}<\hat{\rho}_{i i}^{\sigma}>\left|\phi_{i}(\vec{r})\right|^{2}+\sum_{\sigma} \sum_{i \neq j}^{N}<\hat{\rho}_{i j}^{\sigma}>\phi_{i}^{*}(\vec{r}) \phi_{j}(\vec{r})
\end{aligned}
$$

A calculation containing the pair-distribution function involves wave function integrals between different sites, which is related to the overlap of two electronic wave functions. As a first order approximation and without loss generality, the overlap are not considered, so the second terms in (5) and (6) could be omitted in this study. Therefore, the pair-distribution function $g\left(\vec{r}, \overrightarrow{r^{\prime}}\right)$ in (2) becomes

$$
g_{\sigma \sigma^{\prime}}\left(\vec{r}, \overrightarrow{r^{\prime}}\right)=\frac{\sum_{i k(i \neq k)}^{N}<\hat{\rho}_{i i}^{\sigma} \hat{\rho}_{k k}^{\sigma^{\prime}}>\left|\phi_{i}(\vec{r})\right|^{2}\left|\phi_{k}\left(\overrightarrow{r^{\prime}}\right)\right|^{2}}{\sum_{\sigma i}<\hat{\rho}_{i i}^{\sigma}>\left|\phi_{i}(\vec{r})\right|^{2} \sum_{\sigma k}<\hat{\rho}_{k k}^{\sigma}>\left|\phi_{k}\left(\overrightarrow{r^{\prime}}\right)\right|^{2}}
$$

with $g\left(\vec{r}, \overrightarrow{r^{\prime}}\right)=\sum_{\sigma \sigma^{\prime}} g\left(\vec{r}, \overrightarrow{r^{\prime}}\right)$ and $\rho_{i j}^{\sigma}=<a_{i \sigma}^{\dagger} a_{j \sigma}>$.

\section{Approximation approach to the correlation energy}

In the local density approximation (LDA) the exchange-correlation energy can be written, in terms of the pair-distribution function $\widetilde{g}\left(\vec{r}, \overrightarrow{r^{\prime}}\right)$ in the form ${ }^{30,31}$

$$
E_{x c}[\rho]=\frac{e^{2}}{2} \int d^{3} r d^{3} r^{\prime} \rho(\vec{r}) \frac{\left[\widetilde{g}\left(\vec{r}, \overrightarrow{r^{\prime}}\right)-1\right]}{\left|\vec{r}-\overrightarrow{r^{\prime}}\right|} \rho\left(\overrightarrow{r^{\prime}}\right)
$$

where

$$
\widetilde{g}\left(\vec{r}, \overrightarrow{r^{\prime}}\right)=\int_{0}^{1} d \lambda g\left(\vec{r}, \overrightarrow{r^{\prime}} ; \lambda\right)
$$

Here $\widetilde{g}\left(\vec{r}, \overrightarrow{r^{\prime}}\right)$ includes the exchange-correlation information between two spatial points $\vec{r}$ and $\overrightarrow{r^{\prime}}$. Here $\lambda$ means the charge $e^{2}$ in Coulomb interaction is replaced by $\lambda e^{2}$ in the process of calculation. In $(8), \rho\left(\overrightarrow{r^{\prime}}\right)\left[\tilde{g}\left(\vec{r}, \overrightarrow{r^{\prime}}\right)-1\right]$ describes the depletion in density due to the exchange-correlation hole around an electron at position $\vec{r}$. The density depletion due to the exchange-correlation hole around an electron corresponds to exactly one particle, independent of the size of the electron-electron coupling strength. Then we have the sum rule satisfied by the exchange-correlation hole:

$$
\int d^{3} \overrightarrow{r^{\prime}} \rho\left(\overrightarrow{r^{\prime}}\right)\left[\tilde{g}\left(\vec{r}, \overrightarrow{r^{\prime}}\right)-1\right]=-1
$$

It is well known that it is rather difficult to compute the integration in the relation (8) analytically due to the pair-distribution function $\tilde{g}\left(\vec{r}, \overrightarrow{r^{\prime}}\right)$.

Since the HF approximation has already contained the contribution from the exchange effect between two electrons with the same spins, the correlation energy may be obtained by subtracting the HF pair-distribution function $g_{\sigma \sigma}^{H F}\left(\vec{r}, \overrightarrow{r^{\prime}}\right)$ from the exchange-correlation energy $E_{x c}{ }^{28,32}$,

$$
E_{c}\left[\rho_{\uparrow}, \rho_{\downarrow}\right]=\frac{e^{2}}{2} \sum_{\sigma \sigma^{\prime}} \int d^{3} r d^{3} r^{\prime} \frac{\rho_{\sigma}(\vec{r}) \rho_{\sigma \sigma^{\prime}}^{c}\left(\vec{r}, \overrightarrow{r^{\prime}}\right)}{\left|\vec{r}-\overrightarrow{r^{\prime}}\right|}
$$

where $\rho_{\sigma \sigma^{\prime}}^{c}$ is the spin-dependent correlation hole and given by

$$
\rho_{\sigma \sigma^{\prime}}^{c}\left(\vec{r}, \overrightarrow{r^{\prime}}\right)=\rho_{\sigma^{\prime}}\left(\overrightarrow{r^{\prime}}\right)\left[\widetilde{g}_{\sigma \sigma^{\prime}}\left(\vec{r}, \overrightarrow{r^{\prime}}\right)-\delta_{\sigma \sigma^{\prime}} \widetilde{g}_{\sigma \sigma^{\prime}}^{H F}\left(\vec{r}, \overrightarrow{r^{\prime}}\right)\right] .
$$

where $\widetilde{g}_{\sigma \sigma^{\prime}}^{H F}\left(\vec{r}, \overrightarrow{r^{\prime}}\right)$ is the pair-distribution function under the HF approximation, and is given by

$$
\widetilde{g}_{\sigma \sigma^{\prime}}^{H F}\left(\vec{r}, \overrightarrow{r^{\prime}}\right)=\int_{0}^{1} d \lambda g_{\sigma \sigma^{\prime}}^{H F}\left(\vec{r}, \overrightarrow{r^{\prime}} ; \lambda\right)
$$


The difference between the two pair-distribution functions $\left(\widetilde{g}-\widetilde{g}^{H F}\right)$ has to do with the electron correlation. The expression (11) now can be regarded as an integral between the $\pi$ electron at the position $\vec{r}$ and the charge cloud of the spin-dependent correlation hole around the position $\overrightarrow{r^{\prime}}$ of the other $\pi$ electron. Thus the expression (11) can be rewritten as

$$
E_{c}=\frac{1}{2} \sum_{\sigma \sigma^{\prime}} \int d^{3} r d^{3} r^{\prime} \rho_{\sigma}(\vec{r}) v\left(\vec{r}-\overrightarrow{r^{\prime}}\right) \rho_{\sigma \sigma^{\prime}}^{c}\left(\vec{r}, \overrightarrow{r^{\prime}}\right)
$$

where $v\left(\vec{r}-\overrightarrow{r^{\prime}}\right)=\frac{e^{2}}{\left|\vec{r}-\vec{r}^{\prime}\right|}$, the Coulomb interaction between two electrons at the positions $\vec{r}$ and $\overrightarrow{r^{\prime}}$.

In order to complete the calculation of the integration of $E_{c}$, an approximation is needed to simplify the expression for $E_{c}$. In the study of polyacetylene (PA) oligomer, two-electron interaction integral was approximated by abstracting the Coulomb interaction $v\left(\vec{r}-\overrightarrow{r^{\prime}}\right)$ out of the integrand of the many-centered Coulomb integral, and the results showed that the approximation is reasonable ${ }^{33}$. According to that spirit, we may take $v\left(\vec{r}-\overrightarrow{r^{\prime}}\right)$ out of the integrand in (14). For simplicity, in this study only consider the nearest-neighbor electron-electron interaction between two adjacent carbon atom sites are considered, that is, $v\left(\vec{r}-\vec{r}^{\prime}\right)=\lambda v$, where $\lambda$ is due to the replacement of the charge $e^{2}$ in the Coulomb interaction. In this way the expression for $E_{c}$ may become

$$
\begin{aligned}
E_{c} & \approx \frac{\lambda v}{2} \sum_{\sigma \sigma^{\prime}} \int d^{3} r d^{3} r^{\prime} \rho_{\sigma}(\vec{r}) \rho_{\sigma \sigma^{\prime}}^{c}\left(\vec{r}, \vec{r}^{\prime}\right) \\
& =\frac{\lambda v}{2} \sum_{\sigma \sigma^{\prime}} \int d^{3} r d^{3} r^{\prime} \rho_{\sigma}(\vec{r}) \rho_{\sigma^{\prime}}\left(\overrightarrow{r^{\prime}}\right)\left[\widetilde{g}_{\sigma \sigma^{\prime}}\left(\vec{r}, \overrightarrow{r^{\prime}}\right)-\delta_{\sigma \sigma^{\prime}} \widetilde{g}_{\sigma \sigma^{\prime}}^{H F}\left(\vec{r}, \overrightarrow{r^{\prime}}\right)\right] \\
& =\frac{\lambda v}{2}\left[\sum_{\sigma \sigma^{\prime}} \int d^{3} r d^{3} r^{\prime} \rho_{\sigma}(\vec{r}) \rho_{\sigma^{\prime}}\left(\overrightarrow{r^{\prime}}\right) \widetilde{g}_{\sigma \sigma^{\prime}}\left(\vec{r}, \vec{r}^{\prime}\right)-\sum_{\sigma} \int d^{3} r d^{3} r^{\prime} \rho_{\sigma}(\vec{r}) \rho_{\sigma}\left(\overrightarrow{r^{\prime}}\right) \widetilde{g}_{\sigma \sigma}^{H F}\left(\vec{r}, \overrightarrow{r^{\prime}}\right)\right]
\end{aligned}
$$

Remarkably, it is not easy to calculate it without further approximation. According to the sum rule (10), we have

$$
\sum_{\sigma \sigma^{\prime}} \int d^{3} r d^{3} r^{\prime} \rho_{\sigma}(\vec{r}) \rho_{\sigma^{\prime}}\left(\overrightarrow{r^{\prime}}\right) \widetilde{g}_{\sigma \sigma^{\prime}}\left(\vec{r}, \overrightarrow{r^{\prime}}\right)=\frac{1}{2} \sum_{\sigma \sigma^{\prime}} \int d^{3} r d^{3} r^{\prime} \rho_{\sigma}(\vec{r}) \rho_{\sigma^{\prime}}\left(\overrightarrow{r^{\prime}}\right)-\frac{1}{2} \sum_{\sigma} \int d^{3} r \rho_{\sigma}(\vec{r})
$$

Then the expression for $E_{c}$ can be reduced to

$$
E_{c}=\frac{\lambda v}{2}\left[-\frac{1}{2} \sum_{\sigma} \int d^{3} r \rho_{\sigma}(\vec{r})+\frac{1}{2} \sum_{\sigma \sigma^{\prime}} \int d^{3} r d^{3} r^{\prime} \rho_{\sigma}(\vec{r}) \rho_{\sigma^{\prime}}\left(\overrightarrow{r^{\prime}}\right)-\sum_{\sigma} \int d^{3} r d^{3} r^{\prime} \rho_{\sigma}(\vec{r}) \rho_{\sigma}\left(\overrightarrow{r^{\prime}}\right) \widetilde{g}_{\sigma \sigma}^{H F}\left(\vec{r}, \overrightarrow{r^{\prime}}\right)\right] .
$$

Now the remaining central task is to calculate the Hartree-Fock pair-distribution function $g^{H F}\left(\vec{r}, \vec{r}^{\prime}\right)$. From $(7)$, we have

$$
g_{\sigma \sigma}^{H F}\left(\vec{r}, \overrightarrow{r^{\prime}}\right)=\frac{\sum_{i k(i \neq k)}^{N}<\Phi_{H F}\left|\hat{\rho}_{i i}^{\sigma} \hat{\rho}_{k k}^{\sigma}\right| \Phi_{H F}>\left|\phi_{i}(\vec{r})\right|^{2}\left|\phi_{k}\left(\overrightarrow{r^{\prime}}\right)\right|^{2}}{\sum_{i \sigma}^{N}<\Phi_{H F}\left|\hat{\rho}_{i i}^{\sigma}\right| \Phi_{H F}>\left|\phi_{i}(\vec{r})\right|^{2} \sum_{k \sigma}^{N}<\Phi_{H F}\left|\hat{\rho}_{k k}^{\sigma}\right| \Phi_{H F}>\left|\phi_{k}\left(\overrightarrow{r^{\prime}}\right)\right|^{2}} .
$$

where $\mid \Phi_{H F}>$ is the HF ground state.

Inserting (3) and (18) into the expression of $E_{c}$, it yields

$$
E_{c}=\frac{\lambda v}{2}\left[-\frac{1}{2} \sum_{i \sigma} \rho_{i i}^{\sigma}+\frac{1}{2} \sum_{i k, \sigma \sigma^{\prime}}^{i \neq k} \rho_{i i}^{\sigma} \rho_{k k}^{\sigma^{\prime}}-\sum_{i k, \sigma}^{i \neq k}<\Phi_{H F}\left|\hat{\rho}_{i i}^{\sigma} \hat{\rho}_{k k}^{\sigma}\right| \Phi_{H F}>\right] .
$$

The third term in the above expression can be evaluated and it equals

$$
\begin{aligned}
\sum_{\sigma}<\Phi_{H F}\left|\hat{\rho}_{i i}^{\sigma} \hat{\rho}_{k k}^{\sigma}\right| \Phi_{H F}> & =\sum_{\sigma \sigma^{\prime}}<\Phi_{H F}\left|a_{i \sigma}^{\dagger} a_{i \sigma} a_{k \sigma}^{\dagger} a_{k \sigma}\right| \Phi_{H F}> \\
& =\sum_{\sigma}\left[<a_{i \sigma}^{\dagger} a_{i \sigma}><a_{k \sigma}^{\dagger} a_{k \sigma}>-<a_{i \sigma}^{\dagger} a_{k \sigma}><a_{k \sigma}^{\dagger} a_{i \sigma}>\right] \\
& =\sum_{\sigma}\left[\rho_{i i}^{\sigma} \rho_{k k}^{\sigma}-\left(\rho_{i k}^{\sigma}\right)^{2}\right]
\end{aligned}
$$

Here the symmetry is used that $<a_{i \sigma}^{\dagger} a_{k \sigma}>=<a_{k \sigma}^{\dagger} a_{i \sigma}>$. Finally the correlation energy obtained is

$$
E_{c}=\frac{\lambda v}{2}\left[-\frac{1}{2} \sum_{i \sigma} \rho_{i i}^{\sigma}+\sum_{i k \sigma}^{i \neq k}\left(\rho_{i k}^{\sigma}\right)^{2}\right]
$$

This expression (21) is the central result in this paper and it gives the correlation energy for an open or closed one-dimensional $\pi$ electronic system in real space. The correlation energy per electron is $\epsilon_{c}=E_{c} / N, N$ is the total numbers of the carbon atoms in a $\pi$ electron system. For a half filled system, the number of atomic sites and the number of electrons are equal. This relation tells us that when the averages of the bond charge and the electron density at the site $i$ are known, the correlation energy can be evaluated. 


\section{Calculation and results}

In this study, the main consideration is about the correlation energy of the $\pi$ electron systems. For the $\pi$ conjugated polymers, the Hamiltonian of the system is the SSH-type Hamiltonian, $H_{0}$, plus the electron-electron interaction term,

$$
\begin{gathered}
H=H_{0}+\frac{1}{2} \sum_{i j \sigma \sigma^{\prime}} v\left(\vec{r}-\overrightarrow{r^{\prime}}\right) a_{i \sigma}^{\dagger} a_{i \sigma} a_{j \sigma^{\prime}}^{\dagger} a_{j \sigma^{\prime}} \\
H_{0}=\sum_{i j \sigma \sigma^{\prime}} t_{i j}\left(a_{i \sigma}^{\dagger} a_{j \sigma}+\text { h.c. }\right)
\end{gathered}
$$

where $a_{i \sigma}^{\dagger}\left(a_{j \sigma}\right)$ is the creation (annihilation) operator of an $\pi$ electron at the site $i(j)$ with spin $\sigma . v\left(\vec{r}-\overrightarrow{r^{\prime}}\right)$ is the electronelectron interaction, and $\vec{r}\left(\overrightarrow{r^{\prime}}\right)$ means the position vector of an $\pi$ electron at the site $i(j)$. $t_{i j}$ is the hopping term. For an bond-alternated chain, $t_{i, i+1}=t_{0}+(-1)^{i} \delta t_{0}$ with $t_{0}$ being the hopping integral without dimerization and $\delta t_{0}$ being the magnitude of the dimerization due to Peierls transition.

For the one-dimensional $\pi$ electron conjugated polymers with $\mathrm{N}$ carbon atoms such as the bond-alternated chain PA, in the bond order wave (BOW) phase, the average charge density at the site $i \rho_{i i}^{\sigma}=1 / 2$, and the average of the bond charge density $\rho_{i k}^{\sigma}=\rho_{k i}^{\sigma}=<a_{i \sigma}^{\dagger} a_{k \sigma}>$ (here $\left.k=i+1\right)$. Dropping the spin index because of $\rho_{i k}^{\sigma}=\rho_{i k}^{\bar{\sigma}}=\rho_{i k}$, we have $\rho_{i i+1}=\bar{\rho}+(-1)^{i} \delta \rho$. Then (21) becomes

$$
\begin{aligned}
E_{c} & =\frac{\lambda v}{2}\left[-\frac{N}{2}+2 \sum_{i} \rho_{i, i+1}^{2}\right] \\
& =\frac{\lambda v}{2}\left[-\frac{N}{2}+2 \sum_{m=1}^{N / 2}\left(\rho_{2 m-1,2 m}^{2}+\rho_{2 m, 2 m+1}^{2}\right)\right] .
\end{aligned}
$$

Since $\rho_{2 m-1,2 m}^{2}=(\bar{\rho}-\delta \rho)^{2}=\bar{\rho}^{2}-2 \bar{\rho} \delta \rho+(\delta \rho)^{2}$ and $\rho_{2 m, 2 m+1}^{2}=(\bar{\rho}+\delta \rho)^{2}=\bar{\rho}^{2}+2 \bar{\rho} \delta \rho+(\delta \rho)^{2}$, so the correlation energy for PA may be expressed as

$$
E_{c}=-N \frac{\lambda v}{2}\left[\frac{1}{2}-2\left(\bar{\rho}^{2}+(\delta \rho)^{2}\right)\right]
$$

where $N$ is the carbon atom numbers in the system. The correlation energy per $\pi$ electron is then given by the integral over the parameter $\lambda$ from 0 to 1 ,

$$
\epsilon_{c}=-\frac{v}{2}\left\{\int_{0}^{1} \frac{\lambda}{2} d \lambda-2 \int_{0}^{1}\left[\lambda(\bar{\rho}(\lambda))^{2}+\lambda(\delta \rho(\lambda))^{2}\right] d \lambda\right\}
$$

Here $\bar{\rho}(\lambda)$ and $\delta \rho(\lambda)$ are given by the first and second elliptic integrals ${ }^{34}$

$$
\begin{gathered}
\bar{\rho}(\lambda)=\frac{1}{\pi\left(1-z(\lambda)^{2}\right)}\left[E\left(\sqrt{1-z(\lambda)^{2}}\right)-z(\lambda)^{2} K\left(\sqrt{1-z(\lambda)^{2}}\right)\right] \\
\delta \rho(\lambda)=\frac{z(\lambda)}{\pi\left(1-z(\lambda)^{2}\right)}\left[K\left(\sqrt{1-z(\lambda)^{2}}\right)-E\left(\sqrt{1-z(\lambda)^{2}}\right)\right]
\end{gathered}
$$

where $z(\lambda)=\delta t / t$ and the parameters $t$ and $\delta t$ are determined by the relations

$$
\begin{gathered}
t=t_{0}+\lambda v \bar{\rho}_{0} \\
\delta t=\delta t_{0}+\lambda v \delta \rho_{0}
\end{gathered}
$$

where $\bar{\rho}_{0}$ and $\delta \rho_{0}$ are the average charge density and the change of the charge density without the Coulomb interactions.

For the PA, $t_{0}=2.5 \mathrm{eV}$ and $\delta t_{0}=2 \alpha u_{0}=0.269 \mathrm{eV}$, where $\alpha=4.1 \mathrm{eV} / \AA$ and the dimerization $u_{0}=0.0328 \AA^{27}$. In calculation, the lang-range interaction $v$ is $2.4 \mathrm{eV}^{27}$. Table 1 lists the values for different $\lambda$ when using the the relations (29) and (30) to calculate the average of the bond charge density $\bar{\rho}$ and its change $\delta \rho$ from the relations (27) and (28). Putting the resulting $\bar{\rho}$ and $\delta \rho$ into the (26) and integrating over $\lambda$ from 0 to 1 , the correlation energy is then obtained. In Table 1 $I(\lambda)=(\bar{\rho}(\lambda))^{2}+(\delta \rho(\lambda))^{2}$, and $I=2 \int_{0}^{1}\left[\lambda(\bar{\rho}(\lambda))^{2}+\lambda(\delta \rho(\lambda))^{2}\right] d \lambda$. Table 2 lists the values of the correlation energies for PE and PA for contributions from the on-site Coulomb interaction and the nearest-neighbor Coulomb interaction, and also lists the band energy gap $E_{g}^{\prime}$ in this study and that from Ref.27. Fig.1 shows the correlation energy vs the dimerization $\delta t_{0}$.

To see the influence of the parameter $\lambda$ on the the bond charge density and finally on the correlation energy, I calculate the $\bar{\rho}(\lambda)$ and $\delta \rho(\lambda)$ (see the lines from 2nd to 11th in the Table 1). It is seen from the Table 1 that the differences about the various quantities when $\lambda=0$ and when $\lambda=1$ are very small: $\bar{\rho}(0)-\bar{\rho}(1)=0.0026,|\delta \rho(0)-\delta \rho(1)|=0.0200,|I(0)-I(1)|=0.0024$. 
Table 1: To see the influence of the parameter $\lambda$ on the the bond charge density and finally on the correlation energy, the $\bar{\rho}(\lambda)$ and $\delta \rho(\lambda)$ are calculated (see the lines from 2 nd to 11 th in the Table). It is seen that the differences about the various quantities when $\lambda=0$ and when $\lambda=1$ are very small. $t_{0}=2.5, u_{0}=0.0328, \alpha=4.1 \mathrm{eV} / \AA$ and $v=2.4$ for PA. The unit is $\mathrm{eV}$.

\begin{tabular}{cccccccccc}
\hline \hline$z_{0}$ & $\bar{\rho}_{0}$ & $\delta \rho_{0}$ & $\lambda$ & $\mathrm{z}$ & $\bar{\rho}(\lambda)$ & $\delta \rho(\lambda)$ & $I(\lambda)$ & $I$ & $\epsilon_{c}$ \\
\hline \multirow{3}{*}{0.1076} & 0.3144 & 0.0903 & & & & & & & \\
\hline \multirow{3}{*}{0.1076} & 0.3144 & 0.0903 & 0 & 0.1076 & 0.3144 & 0.0903 & 0.1070 & & -0.1716 \\
& & & 0.1 & 0.1129 & 0.3141 & 0.0930 & 0.1073 & & -0.1713 \\
& & & 0.2 & 0.1178 & 0.3138 & 0.0956 & 0.1076 & -0.1709 \\
& & & 0.3 & 0.1225 & 0.3135 & 0.0979 & 0.1079 & -0.1706 \\
& & & 0.4 & 0.1269 & 0.3132 & 0.1001 & 0.1081 & -0.1703 \\
& & & 0.5 & 0.1311 & 0.3130 & 0.1021 & 0.1084 & -0.1700 \\
& & & 0.6 & 0.1351 & 0.3127 & 0.1039 & 0.1086 & -0.1697 \\
& & 0.7 & 0.1389 & 0.3125 & 0.1057 & 0.1088 & -0.1694 \\
& & & 0.8 & 0.1425 & 0.3122 & 0.1073 & 0.1090 & -0.1692 \\
& & & 0.9 & 0.1460 & 0.3120 & 0.1089 & 0.1092 & & -0.1690 \\
& & 1.0 & 0.1492 & 0.3118 & 0.1103 & 0.1094 & & -0.1687 \\
\hline \hline
\end{tabular}

In addition, the differences between $I$ and $I(\lambda)$ are also small: $|I-I(0)|=0.0017 \mathrm{eV},|I-I(1)|=0.0007 \mathrm{eV}$, and $\mid \epsilon_{c}-\epsilon_{c}(\lambda=$ $0)|=0.0020 \mathrm{eV},| \epsilon_{c}-\epsilon_{c}(\lambda=1) \mid=0.0009 \mathrm{eV}$.

The treatment of the pair-distribution function $(9), \widetilde{g}\left(\vec{r}, \vec{r}^{\prime}\right)=\int_{0}^{1} d \lambda g\left(\vec{r}, \overrightarrow{r^{\prime}} ; \lambda\right)$ is as follows. In the formula (9), Coulomb interaction $e^{2}$ in the Coulomb interaction $v\left(\vec{r}-\overrightarrow{r^{\prime}}\right)$ is replaced by $\lambda e^{2}, \lambda$ changes from 0 to 1 . When $v\left(\vec{r}-\overrightarrow{r^{\prime}}\right)$ appears and changes, the electron wave functions follows the changes. Then the electron density distribution $\rho(\vec{r})$ changes, and then $\rho_{i j}$ changes, which may be expressed as $\bar{\rho}(\lambda)$ and $\delta \rho(\lambda)$ (see (27) and (28)). This can be seen through the expressions (29) and (30). Therefore the calculation of $\widetilde{g}\left(\vec{r}, \vec{r}^{\prime}\right)$ is realized actually through the calculation of $\rho(\vec{r})(\lambda)$. Table 1 shows the results of the correlation energies with integration about the parameter $\lambda$ (see the first line in the Table 1 ).

Note that the bond charge density $\rho_{i j}^{\sigma}$ always is less than half and about 0.3 or so, therefore the second term is less than the first term in the formula (21) and the correlation energy is negative. The electron systems to which the formula is suitable is supposed to be $\pi$ conjugated polymers with long chain ( $N$ is very large) with half filled band in the ground state. For a small molecule system such as $H_{2}$, etc, and those without $\pi$ electrons, the formula is not suitable because the molecule like $H_{2}$ is covalent molecules where the charge density gathers between two atoms. Another reason is that the approximation (15) may bring a bigger error about the electron-electron interaction integral if it is applied to small molecules like $H_{2}$ etc. It is also not suitable to the hydrocarbon such as methane molecule that has no $\pi$ electrons. In derivation of the formula, there is no the excited states to be dealt with.

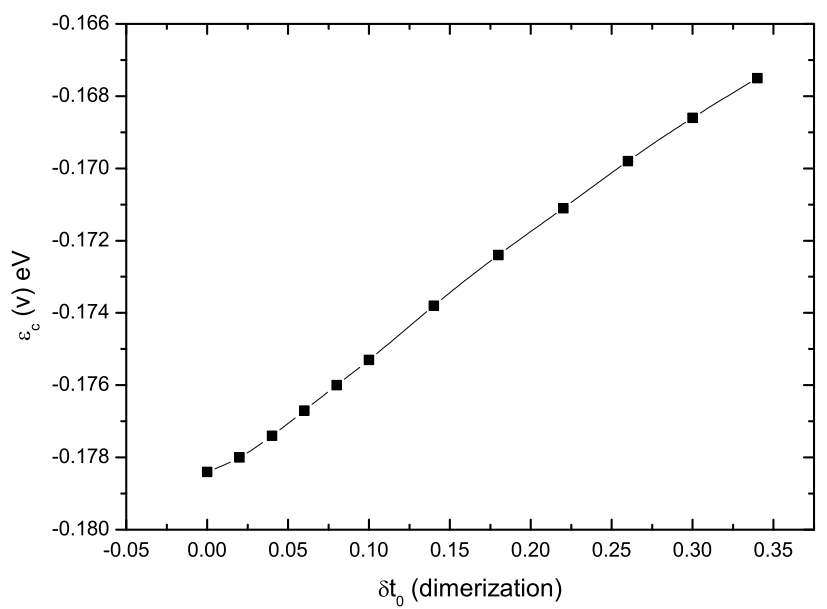

Figure 1: $\epsilon_{c}$ changes with increasing dimerization $\delta t_{0}\left(=2 \alpha u_{0}\right)$ under $v=2.4 \mathrm{eV}$ according to the formula (26). Here $v$ in $\epsilon_{c}(v)$ means the nearest-neighbor Coulomb interaction. 
Table 2: Correlation energies $\epsilon_{c}$ from $n_{i} n_{j}$ and $n_{i \uparrow} n_{i \downarrow}$ for PA and PE. Here $\epsilon_{c}(\mathrm{~V})$ means contribution from the nearest-neighbor Coulomb interaction, $\epsilon_{c}(\mathrm{U})$ means contribution from the on-site Hubbard interaction, and $\epsilon_{c}(\mathrm{U}+\mathrm{V})$ means contribution from both the on-site Hubbard interaction and the nearest-neighbor Coulomb interaction. $E_{g}^{\prime}\left(=E_{g}+\epsilon_{c}\right)$ and $E_{g}^{\prime \prime}$ are the band energy gap. The unit is $\mathrm{eV}$.

\begin{tabular}{ccc}
\hline \hline Systems & $\mathrm{PE}$ & $\mathrm{PA}$ \\
\hline$E_{g}^{\prime}$ & & $1.7832^{*}$ \\
$E_{g}^{\prime \prime}$ & & $1.8^{d}$ \\
\hline & & \\
$\epsilon_{c}(\mathrm{~V})$ & $-0.1725^{a}$ & $-0.1696^{*}$ \\
$\epsilon_{c}(\mathrm{~V})$ & & $-0.1567^{b}$ \\
$\epsilon_{c}(\mathrm{~V})$ & & $-0.0100^{c}$ \\
$\epsilon_{c}(\mathrm{U})$ & $-0.5760^{a}$ & \\
$\epsilon_{c}(\mathrm{U})$ & & $-0.7885^{b}$ \\
$\epsilon_{c}(\mathrm{U})$ & $-0.75^{a}$ & $-0.6208^{c}$ \\
$\epsilon_{c}(\mathrm{U}+\mathrm{V})$ & & $-0.6319^{b}$ \\
$\epsilon_{c}(\mathrm{U}+\mathrm{V})$ & & $-0.6308^{c}$ \\
$\epsilon_{c}(\mathrm{U}+\mathrm{V})$ & & \\
\hline \hline
\end{tabular}

$a$ refers to the reference[22]

$b$ refers to the reference[23]

$c$ refers to the reference[24]

$d$ refers to the reference[27]

* refers to the present study

\section{Discussion}

In the process of derivation for the formula, there are two approximations to be used. One is the tight-bind approximation where the pair-distribution function $g\left(\vec{r}, \overrightarrow{r^{\prime}}\right)$ only involves diagonal elements $\rho_{i i}$ of charge density. Other is that the electron-electron interaction $v\left(\vec{r}-\overrightarrow{r^{\prime}}\right)$ is drawn out of the integrand of the integral expression for $E_{c}$ (See (15)), which was proved to be available in calculating the excitation of the conjugated polymer oligomer ${ }^{33}$. In this way, the correlation energy expression obtained is directly related to the diagonal site charge density $\rho_{i i}^{\sigma}=<a_{i \sigma}^{\dagger} a_{i \sigma}>$ and square of the bond charge density $\rho_{i k}^{\sigma}=<a_{i \sigma}^{\dagger} a_{j \sigma}>(i \neq k)$ that can be calculated directly from the elliptic integrals (27) and (28). This is different from the variational methods ${ }^{22,23,24}$, and also from the the density matrix renormalization group (DMRG) ${ }^{34}$.

If the overlap effect of the $\pi$ electrons at adjacent sites is included, the tight-bind approximation is invalid and the pairdistribution function $g\left(\vec{r}, \vec{r}^{\prime}\right)$ will have both the diagonal terms $\rho_{i i}$ and the off-diagonal terms $\rho_{i j}$ of the charge density. In this case, the electron charge density $\rho_{i i}$ on the sites will be less than half and the bond charge density $\rho_{i j}(i \neq j)$ will be enhanced a little. It may be predictable that when the overlap effects of the $\pi$ electron waves are considered, the correlation energies will become smaller. Moreover, when the overlap integrals are considered, we may discuss the contributions of the correlation energy from the off-diagonal electron-electron interactions ${ }^{35}$.

It is seen from Table 2 that for an infinite polymer PE, the correlation energy was obtained and was $\epsilon_{c}(U+V)=-0.75$ $\mathrm{eV}^{22}$. According to Ref.22, $77 \%$ the correlation energy comes from the contribution of the operator $n_{i \uparrow} n_{i \downarrow}$. Thus the remaining $23 \%$ of the correlation energy comes from the contribution of the long-range Coulomb interaction $V_{i j}(i \neq j)$ or equivalently from the contribution of the operator $n_{i} n_{j}(i \neq j)$. From this, we may have $\epsilon_{c}(V)=0.23 \times(-0.75)=-0.1725 \mathrm{eV}$. The present correlation energy $\left(\epsilon_{c}(V)\right)$ comes from the contribution of the nearest-neighbor Coulomb interaction $(v=2.4 \mathrm{eV})$ between two $\pi$ electrons and is $-0.1696 \mathrm{eV}$. In the Ref.23, the long-range interaction $V$ (here $V=V_{i, i+1}=v$ ) was included within an "effective $U$ " after using some approximation, and the correlation energy $\epsilon_{c}(U+V)$ both including the on-site Hubbard interaction $U$ and the long-range Coulomb interaction $V$ and $\epsilon_{c}(U)$ were obtained for PA, then the correlation energy $\epsilon_{c}(V)$ from the contribution of the long-range Coulomb interaction $V$ may be obtained from the difference between $\epsilon_{c}(U+V)$ and $\epsilon_{c}(U)$ and it yielded $-0.1567 \mathrm{eV}$ with $t_{0}=2.9 \mathrm{eV}$. This value is smaller than the result of the present study. The Ref.24 used the local approach ${ }^{36}$ to calculate the correlation energies. However, the difference between $\epsilon_{c}(U+V)$ and $\epsilon_{c}(U)$ was very small and $\epsilon_{c}(V)=-0.0100$ $\mathrm{eV}$ with $t_{0}=2.5 \mathrm{eV}$ in Ref.24, and this result seems so small.

It is also seen from Table 2 that the present result of the correlation energy $\epsilon_{c}(V)$ for PA is little smaller than that for PE. Both PE and PA includes $s p^{3}$ hybridization. In the calculation of the correlation energy for PE ${ }^{22}$, except $\pi$ electrons between two nearest-neighbor carbon atoms, factors from the different $\pi$ bonds were also taken in numerical computation, so the more correlation effects were included in $\epsilon_{c}(U+V)$ for PE. In addition, the present result $\epsilon_{c}(V)=-0.1696 \mathrm{eV}$ is little larger compared with $\epsilon_{c}(V)=-0.1567 \mathrm{eV}$ from Ref.23. This may be caused by the approximation where the overlap effect between two adjacent $\pi$ electronic wave functions is omitted. When the overlap effect is considered in calculation, the bond charge density will be larger and the site charge density will be less than half, then the result will become smaller.

It is seen from (26) that the correlation energy is an even function of dimerization. The trend of the curve in Fig.1 is kind of quadratic but not complete because there are also the dimerization parameter $z$ in the denominators in the formula. This point can be seen by the following way. Because $z \ll 1$, if we replace $z$ in the denominator in the formula by $z_{0}\left(=\delta t_{0} / t_{0}\right)$, then 
the correlation energy $\epsilon_{c}$ is approximately proportional to $-0.125 v+\frac{A v}{2 \pi^{2}}+\frac{B v}{2 \pi^{2}} z^{2}$, where $A$ and $B$ are the integral constants. At present, there is no similar curve to compare. We may compare the curve in Fig.1 with those in Refs.24 and 27. Because the correlation energy (26) do not contain the contribution form the on-site Hubbard interaction $U$, the curve in Fig. 1 is not completely like those in Fig.2 in Ref.24 and those in Fig.1 in Ref.27 where both $U$ and the long-range interaction were included. Nevertheless, it could be found here that the curve in Fig.1 still have some similar trend with them when the dimerization value is bigger. Though the curve in Fig.2 in Ref.27 was about the ground state energy vs the dimerization, the curve also reflects some information about the correlation energy vs the dimerization because the ground state energy contains the correlation energy contribution in Ref.27.

It is all known that when screening is weak or normal in the $\pi$ electronic conjugated polymers, the electron-electron interaction increases the dimerization and band energy gap ${ }^{35}$. That is, $v$ increases $\delta t_{0}$ and $E_{g}$. In equilibrium state and rigid background, the band energy gap $E_{g}$ of $\mathrm{PA}$ is given by $4 \delta t=4\left(\delta t_{0}+v \delta \rho_{0}\right)$. Under the electron-electron interaction $v$, the average bond charge density $\bar{\rho}_{0}$ decreases slightly with increasing $\delta t_{0}$ and the fluctuation of the bond charge density $\delta \rho_{0}$ increases with increasing $\delta t_{0}$. The decrease of $\bar{\rho}_{0}$ causes the bandwidth (see $t=t_{0}+v \bar{\rho}_{0}$ ) to diminish, and the increase of $\delta \rho_{0}$ make $E_{g}$ increase and at the same time cause $\epsilon_{c}$ (see (25)) to decrease. Then it can be seen from these that $v$ and $\delta \rho_{0}$ are two opposite factors to the correlation energy: $v$ is in favor of the correlation energy but $\delta \rho_{0}$ is a disadvantage to the correlation energy in this study. That is to say, on the one hand, $v$ makes $\delta t_{0}$ and accordingly $\delta \rho_{0}$ increase and then the band energy gap $E_{g}$ increase, on the other hand, the fluctuation $\delta \rho_{0}$ will cause the correlation energy $\epsilon_{c}$ to decrease. As a result, these two opposite influence makes $E_{g}$ decrease from $E_{g}=4 \delta t$ to $E_{g}^{\prime}=E_{g}+\epsilon_{c}$. When $\delta t_{0}=0.269 \mathrm{eV}$ and the corresponding bond charge density $\delta \rho_{0}=0.0903$, we have $E_{g}=1.9429 \mathrm{eV}$ and $\epsilon_{c}=-0.1696 \mathrm{eV}$. Therefore the band energy gap $E_{g}^{\prime}$ containing the nearest-neighbor Coulomb interaction correlation effect becomes $1.7832 \mathrm{eV}$. This value of the band energy gap is close to $E_{g}^{\prime \prime}(1.8 \mathrm{eV})$ obtained by Ab initio computation by author in Ref.27 where the screened interaction was used. These are in qualitative agreement with experiment. In addition, $E_{g}^{\prime}$ increase with increasing dimerization because the correlation energy $\epsilon_{c}$ decreases with dimerization, which is consistent with relation of the band gap and the dimerization.

To my knowledge, there is no similar expressions for the correlation energy only from the nearest-neighbor Coulomb interaction that exists in a simple form at present. In the next study, the more electron-electron interaction terms in the long-range Coulomb interaction will be considered. In addition, although the DMRG is a strong tool to deal with the correlation problems in a many-particle electron system, it is basically a complicated numerical calculation method but not is an analytical expression.

In summary, under the approximation (15) and the tight-bind approximation, a formula (21) of the correlation energy for the long-range (nearest-neighbor) Coulomb interaction $v$ for the conjugated polymers is obtained with the rigid backbone background. Although it is simple, it is direct and effective and easily operational in comparison with other highly involved numerical computation methods including DMRG. The computational result for the correlation energy for PA is available compared to those for PA and PE in different methods ${ }^{22,23}$. The band energy gap $E_{g}^{\prime}$ containing the correlation effect is close to that by ab initio method containing the screening interaction ${ }^{27}$. Because there are no constraints to the systems in the process of the deduction, this relation may be applied to the various $\pi$ electron systems such as $\mathrm{C}_{60}$, benzene rings, and carbon nanotubes, etc.

\section{ACKNOWLEDGMENT}

Special Research Fund (2005) of ChongQing University, ChongQing, P.R. China is acknowledged.

\section{References}

[1] W.Borrmann, P.Fulde, Phys.Rev.B 35, 9569(1987).

[2] D.R.Hatree, Proc.Cambridge Philos.Soc. 24, 89(1928).

[3] V.Fock, Z.Phys.61, 126(1930).

[4] W.Kohn and L.J.Sham, Phys.Rev.140,A 1133(1965).

[5] P.Hohenberg and W.Kohn, Phys.Rev.136 B, 864(1964).

[6] G.D.Mahan and K.R.Subbaswamy, Local Density Theory of Polarizability;

[7] S.F.Boys:Proc.R.Soc.London A 200, 542(1950).

[8] J.Cizek:Adv.Chem.Phys.14,35(1969).

[9] H.Kümmel, K.H.Lührmann, J.G.Zabolitzky: Phys.Lett.C 36, 1(1978).

[10] F.Coester,H.Kümmel:Nucl.Phys.17, 477(1960).

[11] R. Jastrow:Phys.Rev.98, 1479(1955).

[12] J.G.Zabolitzky: In Advances in Nuclear Physics, Vol.12, ed.by W.Negele, E.Vogt(Plenum, Nwe York 1981).

[13] V.R.Pandharipande, R.B.Wiringa: Rev.Mod.Phys. 51, 821(1979). 
[14] H.Mori, Prog.Theor.Phys.33, 423(1965); R.Zwanzig, In Lecture in Theoretcial Physics, Vol.3(Interscience, New York 1961).

[15] L.Hedin: Phys.Rev. A 139, 796(1965).

[16] M. Rohling and S.G. Louie, Phys.Rev.Lett. 82,1959(1999).

[17] R.K.Nesbet, Phys.Rev. 175, 51(1967).

[18] R.K.Nesbet, Phys.Rev.175, 2(1968).

[19] R.K.Nesbet, Phys.Rev.A 3, 87(1971).

[20] G.Stollhoff, P.Vasilopoulos, J.Chem.Phys.84, 2744(1986).

[21] X.Sun, Z.Shuai, K.Naxu, D.L.Lin, and Thomas F.George, Phys.Rev.B 44,11024(1991).

[22] G.König, G.Tollhoff: J.Chem.Phys. 91, 2993(1989).

[23] Dionys Baeriswyl and Kazumi Maki, Phys.Rev.B31,6633(1985).

[24] Peter Horsch, Phys.Rev.B 24, 7351(1981).

[25] G.Stollhoff and P.Fulde, J.Chem.Phys.73, 4548(1980).

[26] P.Horsch and P.Fulde, Z.Phys.B 36, 23(1979).

[27] G.König and G.Stollhoff, Phys.Rev.Lett. 65,1239(1990).

[28] Peter Fulde: Electron Correlations in Molecules and Solds, (Springer-Verlag, Berlin 1993).

[29] O.Gunnarsson, R.O.Jones, J.Chem.Phys.72, 5357(1980).

[30] O.Gunnarsson, B.I.Lundqvist: Phys.Rev.B 13, 4274(1976).

[31] D.C.Langreth, J.P.Perdew: Solid State Common. 17, 1425(1975).

[32] H. Stoll, C.M.E. Pavlidou, H. Preuss: Theor. Chim. Acta 49, 143(1978).

[33] H.Zhao, S.Yokojima,X.Sun,and G.H.Chen,(unpublished);

Also see: WanZhen Liang Satoshi Yokojima, and GuanHuan Chen, J.Chem.Phys. 110, 1844(1999).

[34] S.R. White, Phys.Rev.Lett.69, 2863(1990).

[35] Hua Zhao, Rong-tong Fu, Xin Sun, and Zhi-lin Zhang, Phys. Rev. B 56, 12268(1997).

[36] G.Stollhoff and P.Fulde, Z.Phys.B 26, 251(1977). 